A si mple method for preparing uni form anmor phous fil ms of mxed met al hal i des by means of two- sour ce evapor at $i$ on

\begin{tabular}{|l|l|}
\hline 著者 & KONDO S, TANAKA H, SAl TO T \\
\hline $\begin{array}{l}\text { j our nal or } \\
\text { publ i cat i on t it l e }\end{array}$ & Journal of Physi cs D - Appl i ed Physi cs \\
\hline vol une & 33 \\
\hline number & 22 \\
\hline page range & $2973-2976$ \\
\hline year & $2000-11$ \\
\hline URL & ht t p: //hdl . handl e. net /10098/1545 \\
\hline
\end{tabular}




\title{
A simple method for preparing unitorm amorphous films of mixed metal halides by means of two-source evaporation
}

\author{
S Rondo, H Tanaka and T Sainc \\ Research Center for Development of Far-Infrared Region, Fukui University, Bunkyo, \\ Fukui 910-8507, Japan
}

Abstract. Two-source evaporation of metal halides onto cooled substrates provides a simple method for achieving unifom amorphous networks of solid solution with any composition ratio, yielding film samples. We exemplify its novel use for in situ monitoring of cluster or nanocrystal formation in the films. The method may be applicable to exploring new films heavily doped with clusters or nanocrystals in a controlled manner.

\section{Introduction}

Many investigators have been interested in mesoscopic properties of nanodimensional particles. Various efforts have been made to obtain nanodimensional particles according to the object of investigation. In the study of quantum size effect on excitons, which is one of the most attractive mesoscopic properties exhibited by nanodimensional particles, the particles are usually embedded in a glass or crystal matrix. Such a specimen has mostly been prepared by means of quenching of thermal equilibrium for phase separation of an eutectic mixture. The resulting nanoparticles necessarily have a size and shape distribution, reflecting the phase equilibrium. Furthermore, the mole fraction of the particles is generally very small, typically of the order of $0.1 \mathrm{~mol} \%$ or less. Preparation of a specimen containing nanoparticles of a definite size and shape with a high concentration is not merely desirable for the basic research of their mesoscopic properties, but for their application studies.

On the other hand, it has been shown that halogenides of metals such as $\mathrm{Ag}$ [1], $\mathrm{Cu}$ [2], Tl [3], $\mathrm{Pb}$ [4] or $\mathrm{Cd}$ [5] can be rendered amorphous by quench deposition yielding film samples. The amorphous films exhibit excellent transmittance below the absorption edge and have a well defined, characteristic crystallization temperature at which their absorption spectra drastically change in outline. Many of the crystalline films produced via the amorphous phase show very high transmittance compared with (crystalline) films deposited onto hot substrates (this is particularly the case for lead halides [6]). More interesting is that crystallization of the amorphous films of mixed metal halides produces small clusters or nanocrystals. For instance, we recently obtained [7] $\mathrm{CdI}_{2}$ films in which stable two-molecule $\mathrm{BiI}_{3}$ clusters were densely monodispersed up to $10 \mathrm{~mol} \%$ of $\mathrm{BiI}_{3}$ concentration. The clusters were stoichiometrically embedded in the $\mathrm{Cdl}_{2}$ crystal matrix. It was also possible to disperse CuCl clusters in crystalline $\mathrm{PbCl}_{2}$ films at high density $(\sim 3$ mol\%) [8].

In these studies the amorphous films of the metal halides heavily doped with foreign metal ions were obtainable by evaporating the mixture of two-metal halides (host and guest metal halides) onto cooled substrates; in the amorphous surroundings the cations of the metal halides were replaceable by foreign metal ions up to appreciable concentrations, despite the difference in the valency between the guest and host cations. The crystallization of the amorohous films yielded small clusters or nanocrystals at high densities of the guest-metal halide embedded in the crystalline matrix of the host-metal halide. These results suggest that amorphization and subsequent crystallization of the mixture of metal halides provide a new method for obtaining well identified small clusters or nanocrystals at high densities. These findings motivated the present work.

In order to develop the new method mentioned above, it is of essential importance to facilitate obtaining heavily doped amorphous films with any predetemined doping ratio (it was indeed difficult to obtain such amorphous films by the hitherto employed mixture-evaporation method because of the difference in the vapour pressures between the metal halides contained in the mixture). The main purpose of the present work is to develop a method for obtaining amorphous films of mixed metal halides with any composition ratio. This was achieved by means of simultaneous evaporation of twometal halides using two individual sources of evaporation. As a demonstration, we prepared binary amorphous $\mathrm{TlCl}_{1-x} \mathrm{Br}_{x}$ films using an experimental device previously developed, and confirmed that two-source evaporation is effective in obtaining a uniform amorphous network of mixed metal 


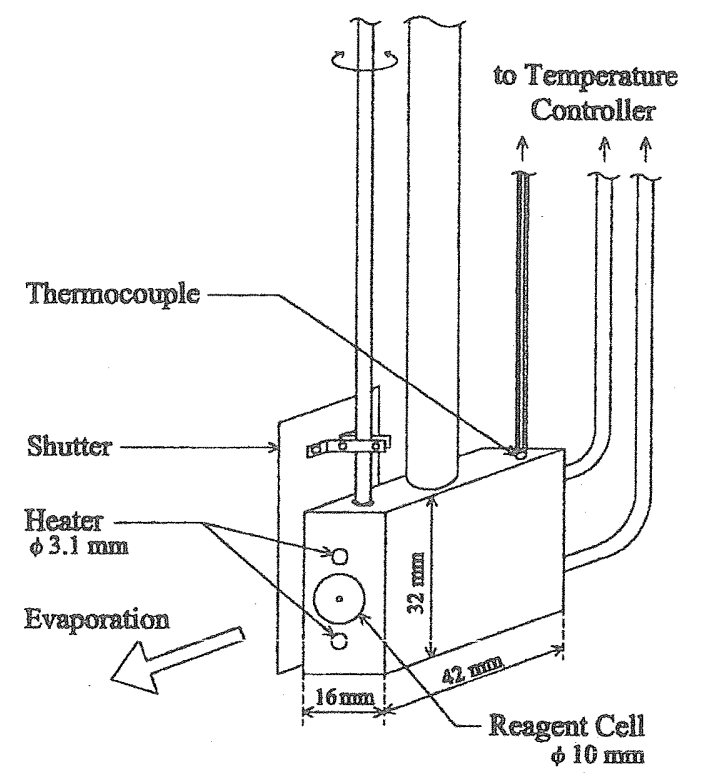

Flgure 1. A sketch of an evaporator used to vaporize metal halide powder reagent. Two evaporators were used in a pair for the purpose of two-source evaporation (see figure 2).

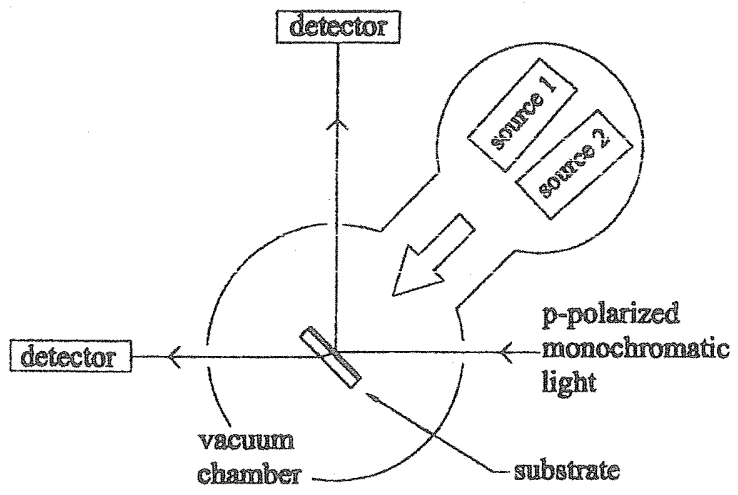

Figure $\mathrm{A}$ schenatic view of the experimental configuration illustrating the preparation of two-source quench deposited amorphous films of mixed metal halides and in situ UV absorption measurement of the films.

halides. To exemplify the potential of the present method, we prepared amorphous $\mathrm{Tl}_{1-x} \mathrm{Cu}_{x} \mathrm{Cl}$ films and monitored their crystallization by in situ exciton spectroscopy.

\section{Expermental details}

Figure 1 is a sketch of the evaporator used for preparing amorphous films. The body of the evaporator is made of a copper block, in which a cylindrical cell (of diameter $10 \mathrm{~mm}$ and length $50 \mathrm{~mm}$ ) with a hole (of diameter $1 \mathrm{~mm}$ ) and two $50 \mathrm{~W}$ cartridge heaters (of diameler $3.1 \mathrm{~mm}$ and length $31.8 \mathrm{~mm}$, from Hakkou Shouji in Japan) are embedded. A powder reagent put in the cell is vaporized in a controlled manner by the heaters. The vapour leads out of the cell through the hole. A rotatable shutter is used to cut off the vapour flow as required.
Figure 2 shows a geometrical configuration of the experimental set-up, in which a two-source (source 1 and source 2) evaporation unit (with a flange) is attached to a vacuum chamber of a cryostat. It is equipped for preparing quench-deposited amorphous films of mixed metal halides with any composition ratio and for studying their crystallization behaviour in situ by optical absorption spectroscopy. First the binary $\mathrm{TICl}_{1-x} \mathrm{Br}_{x}$ system, whose amorphous films were studies previously [9], is used to check the equipment, and then a new, $\mathrm{T}_{1-x} \mathrm{Cu}_{x} \mathrm{Cl}$ system is used to exemplify the potential of the equipment.

Amorphous films of the $\mathrm{TlCl}_{1-x} \mathrm{Br}_{x}$ system were obtained by means of two-source evaporation onto a silicaglass substrate cooled to $77 \mathrm{~K}$ in a vacuum of about $9 \times$ $10^{-6} \mathrm{~Pa}$, using a pair of the above-mentioned evaporators (source 1 for $\mathrm{TlCl}$ and source 2 for $\mathrm{TIBr}$ ) placed $21 \mathrm{~cm}$ in front of the substrate (the distance between the vapour flows at the exits is $22 \mathrm{~mm}$. Films of any concentration ratio were attainable by controlling the temperatures of the evaporators (temperature versus evaporation rate was determined beforehand). The crystallization behaviours of the films were investigated by UV absorption spectroscopy, using an improved double-beam method described in [7]. The method is based on simultaneous measurements of transmittance and reflectance from which accurate optical densities of the films can be determined. The measurements were made in situ for various mixing ratios of the films.

Preparation of amorphous $\mathrm{Tl}_{1-x} \mathrm{Cu}_{x} \mathrm{Cl}$ films was also possible in a similar way, simply by replacing the $\mathrm{TlBr}$ reagent cell of source 2 with a spare cell in which a powder reagent of $\mathrm{CuCl}$ is placed. Crystallization of the films was observed in situ by CuCl-exciton absorption spectroscopy.

\section{Resufts and discussion}

Figure 3 shows absorption spectra (corrected for reflection loss) of a $\mathrm{TICl}_{1-x} \mathrm{Br}_{x}$ film with $x=0.5$. This film was attained by exposing the substrate to steady-state vapour flows of $\mathrm{TlCl}$ (source 1) and $\mathrm{TlBr}$ (source 2), with source temperatures of 385 and $415^{\circ} \mathrm{C}$, respectively (the melting point is $430^{\circ} \mathrm{C}$ for $\mathrm{TlCl}$ and $460^{\circ} \mathrm{C}$ for TIBr). The exposure time was $9 \mathrm{~min}$, leading to a film thickness of about $70 \mathrm{~nm}$ (the growth rate is high enough to insure a sufficiently low water content in the film, considering the background pressure of $9 \times 10^{-6} \mathrm{~Pa}$ ). Spectrum 1 was first measured at $77 \mathrm{~K}$ for the as-deposited (amorphous) film, then the film was annealed at $300 \mathrm{~K}$ for $1 \mathrm{~min}$ and cooled again to $77 \mathrm{~K}$ to measure spectrum 2. Subsequent annealing at $300 \mathrm{~K}$ for $3 \mathrm{~h}$ resulted in spectrum 3 at $77 \mathrm{~K}$. The heating and cooling rates were $\sim 20$ and $\sim 40 \mathrm{~K} \mathrm{~min}^{-1}$, respectively. Spectrum 1, which is characterized by the missing exciton absorption, was shown to obey the Tauc law [10] below $3.78 \mathrm{eV}$, similar to amorphous $\mathrm{TlCl}$ and $\mathrm{TlBr}[9]$, indicating that the film was in the amorphous state (amorphous solid solution). In spectra 2 and 3 there arose exciton transitions characteristic of the crystalline solid solution of the film. (The blue-shifted (by $43 \mathrm{meV}$ ) exciton peak for spectrum 2 compared to that for spectrum 3 is due to the quantum size effect on the exciton as observed for $\mathrm{TlCl}$ [11].)

Previously we showed [9] that uniform amorphous networks of a mixture of TlCl and TIBr are attainable for 


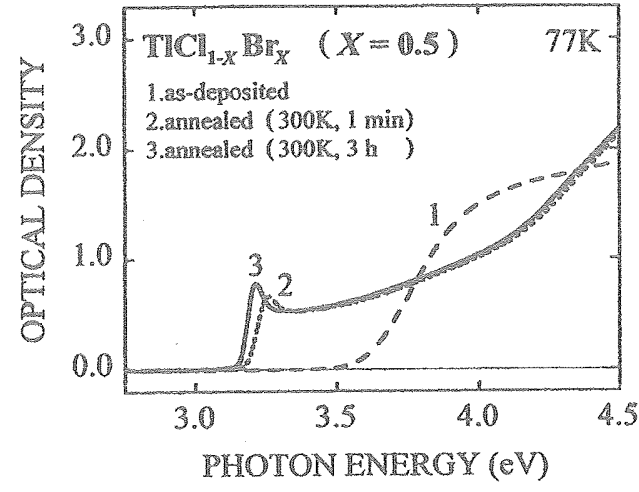

Figures 5 . Absomption spectra of a quench-deposited amorphous firm of mixed $T \mathrm{TCl}_{1-x} \mathrm{TP}_{x}$ solid solution with $x=0.5$, prepared by means of two-source evaporation onto the substrate at $77 \mathrm{~K}$. "The Alm thickness

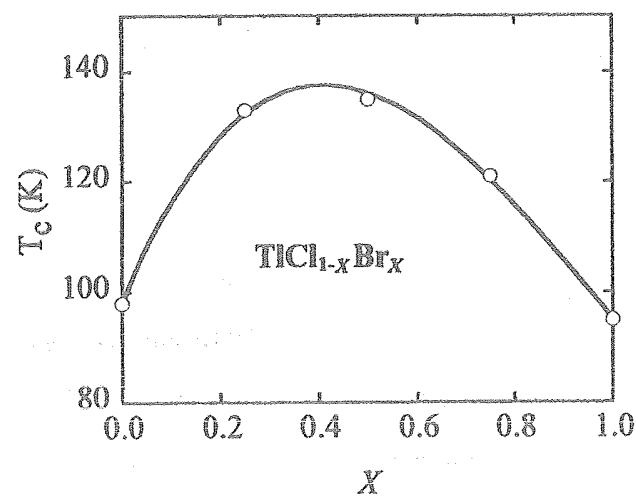

wgerpe 4 Crystalization cemperature $\left(T_{c}\right)$ versus $x$ of amorphous $\operatorname{TCl}_{1-x} \mathrm{Br}_{x}$ fims.

any composition ratio by the method of (single-source) evaporation (onto silica-glass substrates at $77 \mathrm{~K}$ ) of the mixture. These networks have well defined characteristic crystallization temperatures, depending on the composition ratio. To check the quality of the present amorphous films, we traced thermally-induced crystallization of the films in the same way as that employed in [9]. For example, crystallization of the film with $x=0.5$ started to occur at $135.2 \mathrm{~K}\left(T_{c}=135.2 \mathrm{~K}\right)$ and was completed at $138.4 \mathrm{~K}$ under heating at a rate of $1 \mathrm{~K} \mathrm{~min}^{-1}$. The crystallization temperature $T_{c}$ versus the TIBr concentration $x$, ploted in figure 4 , is in good agreement with the previous result (chemical disorder due to making the solid into a solution has the effect of increasing the nucleation activation energy of the amorphous state). Therefore, it is considered that uniform amorphous networks were achieved for any composition ratio by the present two-source evaporation method.

Figure 5 shows absorption spectra of $\mathrm{T}_{1-x} \mathrm{Cu} x \mathrm{Cl}$ film with $x=0.4$. Spectrum 1 was first measured at $77 \mathbb{K}$ for the as-prepared film. Then the fim was subjected to various annealing cycles in order to observe irreducible change in the spectrum at $77 \mathrm{~K}$ with increasing annealing temperature. Heating and cooling in each annealing cycle were performed at rates of 1 and $10 \mathrm{~K} \mathrm{~min}^{-1}$, respectively. The annealing temperature, $T_{a}(K)$, and annealing time, $t_{a}$

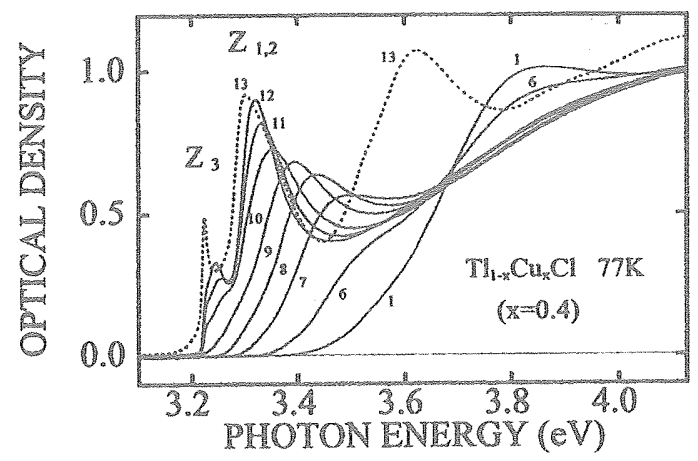

Figumes. The effect of annealing on optical absorption of a $\mathrm{Tl}_{1-x} \mathrm{Cu}_{x} \mathrm{Cl}(x=0.4)$ fim prepared by means of two-source evaporation onto the substrate at $77 \mathrm{~K}$. The absorption spectra are measured at $77 \mathrm{~K}$ for increasing annealing temperatures (see text).

(min), for each spectrum labelled with the number $n$ are, in terms of $n\left(T_{a}, \hat{t}_{a}\right)$, as follows: $2(100,1), 3(120,1)$, $4(140,1), 5(160,1), 6(180,1), 7(200,1), 8(220,1), 9(240,1)$, $10(260,1), 11(280,1), 12(300,1), 13(300,10), 14(300,180)$ and $15(360,1)$; spectra $2-5$ and spectra 14 and 15 are not shown in the figure because their shapes are entirely the same as spectrum 5 and spectrum 13 , respectively. In the range of annealing temperature, $140 \mathrm{~K}<T_{a}<300 \mathbb{K}$, a gradual change occurs in the spectral structure with increasing $T_{a}$ : a new absorption band shows up at arownd $3.5 \mathrm{eV}$ for $\mathbb{T}_{a}=180 \mathrm{~K}$ (spectnum 6). As the annealing temperature is increased, it becomes prominent and shifts to low energies exhibiting two absorption bands, at arownd 3.24 and $3.32 \mathrm{eV}$ for $T_{a}=300 \mathrm{~K}\left(t_{a}=1 \mathrm{~min}\right.$, specinum 12), which correspond to the CuCl $\mathbb{Z}_{3}$ and $\mathbb{Z}_{1,2}$ excitons [12], respectively. As compared to this gradual change, further annealing at $300 \mathrm{~K}$, but only for 10 min, of the film (spectrum 13 ) gives rise to an abrupt spectral change characterized by the sharpened $Z_{3}$ exciton peak and a new, strong absorption band appearing around $3.6 \mathrm{eV}$ with a weak shonlder at about $3.53 \mathrm{eV}$. These features of spectrum 13 remain unchanged on further annealing (spectra 14 and 15 , not shown in the figure).

As mentioned in section 1, amorphous films of metal halides, including $\mathrm{TlCl}$ and $\mathrm{CuCl}$, have well defined crystallization temperatures ( $98 \mathrm{~K}$ for TICl [3], $177 \mathrm{~K}$ for $\mathrm{CuCl}$ (21), at which a distinct change in their absorption spectrum occurs. Such a characteristic change in the specirum also occurs in the $\mathrm{Tl}_{1-x} \mathrm{Cu}_{x} \mathrm{Cl}$ film for $\mathrm{T}_{a}=300 \mathrm{~K}$, as seen from the distinct difference between spectra 12 and 13 in figure 5. It may be supposed, therefore, that the fhrm is in the amorphous state below $300 \mathrm{~K}$ and crystallization of the flun starts to occur at $T_{a}=300 \mathrm{~K}$ and is completed within 10 min at that temperature. We note that spectrum 13 exhibits several fine structures in the measured region above $4 \mathrm{eV}$ (up $106.2 \mathrm{eV}$, though not shown in the figure) reminiscent of van Hove singularities of the associated energy band (while no fine structures are observed in spectrum 12). Completion of the crystallization (within $10 \mathrm{~min}$ at $300 \mathrm{~K}$ ) is evidenced by the fact that no further spectral change occurred during further amealing at $300 \mathrm{~K}$ or even at higher annealing temperatures $(\leqslant 360 \mathrm{~K})$. As for the gradual spectral change below $300 \mathrm{~K}$, it is interesting to note that when the film is annealed at 
temperatures near the crystallization temperature (177 kn) of amorphous $\mathrm{CuCl}$, a new absorption band shows up at around $3.5 \mathrm{eV}$ (spectrum 6). This indicates the formation of $\mathrm{CuCl}$ clusters in the amorphous surroundings. The average radius of the clusters is estimated to be about $1.0 \mathrm{~mm}$ according 10 [13]. Details will be reported elsewhere, including an explanation for the much higher crystallization temperature $(300 \mathrm{~K})$ of the present fim than those of amorphous films of $\mathrm{TlCl}(98 \mathrm{~K})$ and $\mathrm{CuCl}(177 \mathrm{~K})$. These results demonstrate the potential of the present method for exploring a new film heavily doped with clusters or nanocrystals in a controlled manner, as well as for in situ monitoring of cluster or nanocrystal formation in the film.

\section{Conchusion}

We have developed a simple method for preparing uniform quench-deposited amorphous films of mixed metal halides with any composition ratio. It is based on simultaneous evaporation of two metal halides onto a cooled substrate using two individual sources of evaporation. We exemplify its practical use for in situ monitoring of cluster or nanocrystal formation in the film. The method may be applicable to exploring new films heavily doped with clusters or nanocrystals of a definite size and shape in a controlled manner, such as $\mathrm{Cd}_{2}$ fims heavily doped with $\mathrm{Bil}_{3}$ clusters or $\mathrm{PbCl}_{2}$ films heavily doped with $\mathrm{CuCl}$ nanocrystals, mentioned in section 1. Such films are not merely desirable for the basic research of particle confunement problems and related mesoscopic enhancement of linear and nonlinear optical properties, but for their application studies. Sudies on achieving $\mathrm{Bil}_{3}$ hexagon clusters (stable due to high symmetry) densely xnonodispersed in $\mathrm{CdH}_{2}$ films are in progress and the results will be reported in the near future.

The present method of two-source evaporation has only been studied in conjunction with visible to ultraviolet, or exciton, spectroscopy. However, the two-source evaporation unit itself is designed to allow its optional use (it can be used as an attachment for other equipment), It is interesting to apply the method to other optical sudies such as infrared and Raman spectroscopies.

\section{Aclroundedgnents}

This work was partly suppored by the Grant-in-Aid for Scientific Research from the Ministry of Education, Science, Sports and Culure, and also partly by The Hokuriku Industrial Advancement Center, Japan.

\section{Reterences}

[1] See, for example, Gotwald H $\mathrm{G}$ and Weil $\mathrm{K} \mathrm{G} 1988 \mathrm{Ber}$ Bunsenges. Phys. Chem. 9260

[2] See, for example, Kondo S and Seki T1992 Marer. Sci. Eng. 15133

[3] See, for example, Kondo S, Itoh T, Saito T and Mekata M 1991 Solid Siate Commun. 7955

[4] Sce, for example, Kondo S, Sakai $\mathrm{T}$, Tanaka $\mathrm{H}$ and Saito $\mathrm{T}$ 1998 Phys. Rev. B 5911401

[5] See, for example, Kondo $S$, Kagawa $S$ and Saito 1996 Phys. Status Soldi a 15453

[6] See, for example, Kondo $\mathrm{S}$, 1 wh $\mathrm{M}$ and Saito $\mathrm{T} 1996 \mathrm{phys}$. Sicutus Solidi a 1591

[7] Kondo $\$$, Suzuki T and Saito T 1998 J. Phys. D: Appl. Phys. 312733

[8] Kondo $\mathrm{S}$, Tanaka $\mathrm{N}$ and Saito T 2000 J. Phys. D: Appl. Phys. 33327

[9] Kondo S, Yamagishi N and Saito 1998 J. Phys. Soc Japon 6733

1109 Tauc 1, Grigorovici $R$ and Vancu A 1966 phys. Srous Solid: 15627

[11] 筑ondo S and Nakano M 1992 . Phys. Soc. Japan 6 直 4255

$112]$ Kondo S, thoh Tand Mekata M 1991 J. Phys. Soc. Japan 64 3192

[13] Ekimov A I, Eros AIL and Onushchenko 1985 Solid Siate Commun. 56921 